doi:10.13108/2021-13-3-36

\title{
HYPERBOLIC DIFFERENTIAL-DIFFERENCE EQUATIONS WITH NONLOCAL POTENTIALS
}

\author{
N.V. ZAITSEVA
}

\begin{abstract}
We consider a three-parametric set of solutions for a two-dimensional hyperbolic differential-difference equation in a half-plane containing the sum of a differential operator and shift operators with respect to a spatial variable ranging on the entire real axis (or a differential-difference equation with nonlocal potentials). All shifts in potentials with respect to the spatial variable are arbitrary real numbers and no commensurability is assumed. This is the most general case.

Nowadays, elliptic and parabolic functional-differential equations, and, in particular, differential-difference equations, are studied well enough. The aim of this work is to investigate hyperbolic differential-difference equations with shift operators in the space variable, which, as far as we know, have not been studied previously. The nature of the physical problems leading to such equations is fundamentally different from the problems for the classical equations of mathematical physics. To construct solutions, we employ a classical operation scheme is used, according to which the direct and then the inverse Fourier transforms are formally applied to the equation. However, if in the classical case the application of the Fourier transform leads to the study of polynomials with respect to the dual variable, in our case, due to the fact that in the Fourier images a shift operator is a multiplier, the symbol of the differential-difference operator is no longer a polynomial, but a combination of a power function and trigonometric functions with incommensurable arguments. This gives rise to computational difficulties and completely different effects in the solution. Generally speaking, this scheme leads to solutions in the sense of generalized functions. However, in this case it is possible to prove that the obtained solutions are classical.

We prove a theorem that if the real part of the symbol of the differential-difference operator in the spatial variable involved in the equation is positive, then the constructed solutions are classical. Classes of equations for which this condition is satisfied are given. We obtain the relations for the coefficients and shifts in the equation ensuring the required positivity for the real part of the symbol of the differential-difference operator in the equation.
\end{abstract}

Keywords: hyperbolic equation, differential-difference equation, incommensurable shifts, classical solution.

Mathematics Subject Classification: 35R10, 35L10

\section{INTRODUCTION}

First a differential-difference equation appeared in a work by J. Bernoulli [1] in the problem on a tight massless of a finite length, along which equal and equally-spaced masses are distributed. The equation he considered then arose while developing the sound theory and attracted the attention of many other mathematicians, see, for instance [2] and the references therein. Book [3] provides a rich content on the theory of linear differential-difference equations with constant coefficients arising often in the theory of automatic control. In book [4], the

N.V. Zaitseva, Hyperbolic differential-Difference Equations With nonlocal potentials.

(C) Zaitseva N.V. 2021.

The work is supported by the Center of Fundamental and Applied mathematics of MSU.

Submitted February 26, 2021. 
theory of linear differential-difference equations with varying coefficients is presented in details and a lot of attention is paid to an asymptotic behavior of solutions and to the stability theory.

The study of problems in mechanics of continuum media lead the researchers to considering partial differential-difference equations. At present, problems for such equations in bounded domains are rather well studied, see, for instance, [5]-[10] and the references therein. In unbounded domains, problems for parabolic [11] and elliptic differential-difference equations [12]-[16] were studied in details. In particular, works [14], [15] were devoted to strongly elliptic equations with nonlocal potentials in one of spatial variables arising in models of nonlinear optics. Hyperbolic differential-difference equations were earlier studied for the case, when the shift operators in the equation acts in the time [17]. In works [18]-[20] there were considered hyperbolic differentialdifference equations involving superpositions of differential operators and the shift operator in the spatial variable.

Let $a, b_{k}, h_{k}, k=\overline{1, n}$, be given real number. In the half-plane $(x, t) \in \mathbb{R}^{1} \times(0,+\infty)$ we consider a hyperbolic equation involving the sum of a differential operator and shift operators along the spatial variable

$$
\frac{\partial^{2} u(x, t)}{\partial t^{2}}=L u \stackrel{\text { def }}{=} a^{2} \frac{\partial^{2} u(x, t)}{\partial x^{2}}-\sum_{k=1}^{n} b_{k} u\left(x-h_{k}, t\right),
$$

and according the terminology of [3], we call it differential-difference equation. All shifts $h_{k}$, $k=\overline{1, n}$, in the equation are arbitrary quantities.

There are well-known problems in mathematical physics giving rise to classical partial differential equations involving, apart of the derivatives, the unknown function or a potential. An example is the equation of small oscillations of a heavy homogeneous filament with a fixed upper end-point near its vertical equilibirum. When studying electric oscillations in wires, the current equation (or the voltage equation) contains an unknown function, if one does not neglect the losses (leakage) through the insulation of the wires and the magnitude of the resistance. The propagation of electrical oscillations is described by telegraph equations. It is possible to introduce acoustic analogs of resistance and leakage, the friction of the gas against the vessel walls and the porosity of the medium, respectively, and obtain hyperbolic equations with the classical potential.

The solutions of a hyperbolic equation

$$
u_{t t}-a^{2} u_{x x}+c u=0
$$

in which the phase velocity of harmonic waves depends on the frequency, that is, the wave dispersion is described, arise as $c \neq 0$.

The diffusion process of an unstable gas (diffusion with decay) leads to the equation

$$
u_{t}=a^{2} \triangle u+\beta u,
$$

where $\beta<0$ is a characteristics of a material. Diffusion processes under the presence of chain reactions (for example, the process of neutron multiplication) are of great interest; the study of therse processes lead one to the equation

$$
u_{t}=a^{2} \triangle u+\beta u
$$

where $\beta>0$ (diffusion with propagation).

The study of steady-state vibrations (mechanical, acoustic, electromagnetic, etc.) leads to the wave equation

$$
\triangle u+c u=0
$$

where $c>0$. Moreover, there are many problems on steady oscillations in an inhomogeneous media, which are problems in the diffraction theory.

In considered equation (1.1) the potentials are nonlocal since all real shifts $h_{k}, k=\overline{1, n}$, are not infinitesimal and can be arbitrary large. We note that the shift operator is not relatively 
bounded with respect to the differential operator. Equation (1.1) relates the value of the sought function $u$ at $(n+1)$ different points in the half-plane $(x, t) \in \mathbb{R}^{1} \times(0,+\infty)$, and this is the principal difference between differential-difference equations and the classical equations in mathematical physics.

The real part of the symbol of the differential-difference operator $L$ is of form

$$
\operatorname{Re} L(\xi)=-a^{2} \xi^{2}-\sum_{k=1}^{n} b_{k} \cos \left(h_{k} \xi\right) .
$$

The operator $-L(\xi)$ is called positive if $-\operatorname{Re} L(\xi)>0$ for each $\xi \in \mathbb{R}^{1}$, i.e., if the inequality

$$
a^{2} \xi^{2}+\sum_{k=1}^{n} b_{k} \cos \left(h_{k} \xi\right)>0
$$

holds. In what follows we assume that the operator $-L(\xi)$ is positive.

Definition 1.1. A function $u(x, t)$ is called a classical solution of equation (1.1) if at each point of the half-plane $(x, t) \in \mathbb{R}^{1} \times(0,+\infty)$ there exist classical derivatives $u_{t t}$ and $u_{x x}$ defined in terms of the limits of the quotients of finite differences and at each point relation (1.1) is satisfied.

\section{Construction of SOlutions}

In order to find solutions of equation (1.1), we employ a classical Gelfand-Shilov operation scheme [21, Sect. 10], according to which we apply the Fourier transform $F_{x}$ to identity (1.1) and then for the function $\widehat{u}(\xi, t)=F_{x}[u](\xi, t)$ we get an ordinary differential equation

$$
\frac{d^{2} \widehat{u}(\xi, t)}{d t^{2}}=-\left(a^{2} \xi^{2}+\sum_{k=1}^{n} b_{k} e^{i h_{k} \xi}\right) \widehat{u}(\xi, t), \quad \xi \in(-\infty,+\infty) .
$$

Its characteristic equation has the roots

$$
k_{1,2}= \pm i \sqrt{a^{2} \xi^{2}+\sum_{k=1}^{n} b_{k} e^{i h_{k} \xi}}= \pm i \rho(\xi) e^{i \varphi(\xi)},
$$

where

$$
\begin{aligned}
\rho(\xi) & :=\left(\left(a^{2} \xi^{2}+\sum_{k=1}^{n} b_{k} \cos \left(h_{k} \xi\right)\right)^{2}+\left(\sum_{k=1}^{n} b_{k} \sin \left(h_{k} \xi\right)\right)^{2}\right)^{\frac{1}{4}} \\
\varphi(\xi) & :=\frac{1}{2} \arctan \frac{\sum_{k=1}^{n} b_{k} \sin \left(h_{k} \xi\right)}{a^{2} \xi^{2}+\sum_{k=1}^{n} b_{k} \cos \left(h_{k} \xi\right)} .
\end{aligned}
$$

We note that for all real value of the parameters $a, b_{k}, h_{k}, k=\overline{1, n}$ and $\xi$, the functions $\rho(\xi)$ and $\varphi(\xi)$ are well-defined. Thus, the general solution of equation (2.1) is defined by the formula

$$
\begin{aligned}
\widehat{u}(\xi, t) & =C_{1}(\xi) e^{i t \rho(\xi)[\cos \varphi(\xi)+i \sin \varphi(\xi)]}+C_{2}(\xi) e^{-i t \rho(\xi)[\cos \varphi(\xi)+i \sin \varphi(\xi)]} \\
& =C_{1}(\xi) e^{-t \rho(\xi)[\sin \varphi(\xi)-i \cos \varphi(\xi)]}+C_{2}(\xi) e^{t \rho(\xi)[\sin \varphi(\xi)-i \cos \varphi(\xi)]}
\end{aligned}
$$

where $C_{1}(\xi), C_{2}(\xi)$ are arbitrary constants depending on the parameter $\xi$. Letting $C_{1}(\xi)=1$, $C_{2}(\xi)=0$, by the latter identity we have:

$$
\widehat{u}(\xi, t)=e^{-t G_{1}(\xi)} e^{i t G_{2}(\xi)}
$$


where we have introduced the notations

$$
G_{1}(\xi):=\rho(\xi) \sin \varphi(\xi), \quad G_{2}(\xi):=\rho(\xi) \cos \varphi(\xi) .
$$

We apply the inverse Fourier transform $F_{\xi}^{-1}$ to identity $(2.4)$ and we obtain

$$
\begin{aligned}
u(x, t) & =\frac{1}{2 \pi} \int_{-\infty}^{+\infty} e^{-t G_{1}(\xi)} e^{i t G_{2}(\xi)} e^{-i x \xi} d \xi=\frac{1}{2 \pi} \int_{-\infty}^{+\infty} e^{-t G_{1}(\xi)} e^{i\left(t G_{2}(\xi)-x \xi\right)} d \xi \\
& =\frac{1}{2 \pi} \int_{-\infty}^{+\infty} e^{-t G_{1}(\xi)} \cos \left(t G_{2}(\xi)-x \xi\right) d \xi+\frac{i}{2 \pi} \int_{-\infty}^{+\infty} e^{-t G_{1}(\xi)} \sin \left(t G_{2}(\xi)-x \xi\right) d \xi
\end{aligned}
$$

It should be noted that while applying formally the direct Fourier transform $F_{x}$ and the inverse Fourier transform $F_{\xi}^{-1}$, we do not care about the convergence of the integrals in 2.6 in accordance with the scheme in [20, Sect. 10], in which the solutions are treated in the sense of generalized functions. On the base of (2.6) and arguing as in [14], [15], we prove the following statement.

Theorem 2.1. Under condition (1.2), the functions

$$
\begin{aligned}
& F(x, t ; \xi):=e^{-t G_{1}(\xi)} \cos \left(t G_{2}(\xi)-x \xi\right), \\
& H(x, t ; \xi):=e^{-t G_{1}(\xi)} \sin \left(t G_{2}(\xi)-x \xi\right),
\end{aligned}
$$

where $G_{1}(\xi)$ and $G_{2}(\xi)$ are defined by identities (2.5), satisfy equation (1.1) in the classical sense.

Proof. We first substitute function (2.7) into equation (1.1). In order to do this, we find:

$$
\begin{aligned}
F_{x}(x, t ; \xi)= & \xi e^{-t G_{1}(\xi)} \sin \left(t G_{2}(\xi)-x \xi\right) \\
F_{x x}(x, t ; \xi)= & -\xi^{2} e^{-t G_{1}(\xi)} \cos \left(t G_{2}(\xi)-x \xi\right) \\
F_{t}(x, t ; \xi)= & -G_{1}(\xi) e^{-t G_{1}(\xi)} \cos \left(t G_{2}(\xi)-x \xi\right)-G_{2}(\xi) e^{-t G_{1}(\xi)} \sin \left(t G_{2}(\xi)-x \xi\right) \\
F_{t t}(x, t ; \xi)= & \left(G_{1}^{2}(\xi)-G_{2}^{2}(\xi)\right) e^{-t G_{1}(\xi)} \cos \left(t G_{2}(\xi)-x \xi\right) \\
& +2 G_{1}(\xi) G_{2}(\xi) e^{-t G_{1}(\xi)} \sin \left(t G_{2}(\xi)-x \xi\right)
\end{aligned}
$$

In view of 2.5 we have

$$
2 G_{1}(\xi) G_{2}(\xi)=\rho^{2}(\xi) \sin 2 \varphi(\xi) .
$$

Since the variable $\varphi(\xi)$ is defined by expression $(2.3)$, then the inequality $|2 \varphi(\xi)|<\frac{\pi}{2}$ holds and therefore, $\cos 2 \varphi(\xi)>0$. Then the identities

$$
\sqrt{\cos ^{2} 2 \varphi(\xi)}=|\cos 2 \varphi(\xi)|=\cos 2 \varphi(\xi)
$$

hold and the following relation

$$
\begin{aligned}
\sin 2 \varphi(\xi) & =\frac{\tan 2 \varphi(\xi)}{\sqrt{1+\tan ^{2} 2 \varphi(\xi)}} \\
& =\tan \left(\arctan \frac{\sum_{k=1}^{n} b_{k} \sin \left(h_{k} \xi\right)}{a^{2} \xi^{2}+\sum_{k=1}^{n} b_{k} \cos \left(h_{k} \xi\right)}\right)\left(1+\tan ^{2}\left(\arctan \frac{\sum_{k=1}^{n} b_{k} \sin \left(h_{k} \xi\right)}{a^{2} \xi^{2}+\sum_{k=1}^{n} b_{k} \cos \left(h_{k} \xi\right)}\right)\right)^{-\frac{1}{2}}
\end{aligned}
$$




$$
\begin{aligned}
& =\frac{\sum_{k=1}^{n} b_{k} \sin \left(h_{k} \xi\right)}{a^{2} \xi^{2}+\sum_{k=1}^{n} b_{k} \cos \left(h_{k} \xi\right)}\left(1+\frac{\left(\sum_{k=1}^{n} b_{k} \sin \left(h_{k} \xi\right)\right)^{2}}{\left(a^{2} \xi^{2}+\sum_{k=1}^{n} b_{k} \cos \left(h_{k} \xi\right)\right)^{2}}\right)^{-\frac{1}{2}} \\
& =\frac{\sum_{k=1}^{n} b_{k} \sin \left(h_{k} \xi\right)}{a^{2} \xi^{2}+\sum_{k=1}^{n} b_{k} \cos \left(h_{k} \xi\right)}\left(\frac{\left(a^{2} \xi^{2}+\sum_{k=1}^{n} b_{k} \cos \left(h_{k} \xi\right)\right)^{2}}{\left(a^{2} \xi^{2}+\sum_{k=1}^{n} b_{k} \cos \left(h_{k} \xi\right)\right)^{2}+\left(\sum_{k=1}^{n} b_{k} \sin \left(h_{k} \xi\right)\right)^{2}}\right)^{\frac{1}{2}}
\end{aligned}
$$

is true. By condition $(1.2)$ and formula $(2.2)$ the latter identity yields

$$
\sin 2 \varphi(\xi)=\frac{1}{\rho^{2}(\xi)} \sum_{k=1}^{n} b_{k} \sin \left(h_{k} \xi\right),
$$

and this implies that

$$
2 G_{1}(\xi) G_{2}(\xi)=\sum_{k=1}^{n} b_{k} \sin \left(h_{k} \xi\right) .
$$

Under the established inequality $\cos 2 \varphi(\xi)>0$ and condition 1.2 we find:

$$
\begin{aligned}
G_{1}^{2}(\xi)-G_{2}^{2}(\xi) & =\rho^{2}(\xi)\left(\sin ^{2} \varphi(\xi)-\cos ^{2} \varphi(\xi)\right) \\
& =-\rho^{2}(\xi) \cos 2 \varphi(\xi)=-\frac{\rho^{2}(\xi)}{\sqrt{1+\tan ^{2} 2 \varphi(\xi)}} \\
& =-\rho^{2}(\xi)\left(\frac{\left(a^{2} \xi^{2}+\sum_{k=1}^{n} b_{k} \cos \left(h_{k} \xi\right)\right)^{2}}{\left(a^{2} \xi^{2}+\sum_{k=1}^{n} b_{k} \cos \left(h_{k} \xi\right)\right)^{2}+\left(\sum_{k=1}^{n} b_{k} \sin \left(h_{k} \xi\right)\right)^{2}}\right)^{\frac{1}{2}} \\
& =-a^{2} \xi^{2}-\sum_{k=1}^{n} b_{k} \cos \left(h_{k} \xi\right) .
\end{aligned}
$$

In view of the found expressions $G_{1}^{2}(\xi)-G_{2}^{2}(\xi)$ and $2 G_{1}(\xi) G_{2}(\xi)$, by identity 2.9 we get:

$$
\begin{aligned}
F_{t t}(x, t ; \xi)= & -\left(a^{2} \xi^{2}+\sum_{k=1}^{n} b_{k} \cos \left(h_{k} \xi\right)\right) e^{-t G_{1}(\xi)} \cos \left(t G_{2}(\xi)-x \xi\right) \\
& +\sum_{k=1}^{n} b_{k} \sin \left(h_{k} \xi\right) \cdot e^{-t G_{1}(\xi)} \sin \left(t G_{2}(\xi)-x \xi\right) .
\end{aligned}
$$

Substituting the found derivatives $F_{t t}$ and $F_{x x}$ into equation (1.1), we find:

$$
\begin{aligned}
F_{t t}(x, t ; \xi) & -a^{2} F_{x x}(x, t ; \xi) \\
& =-e^{-t G_{1}(\xi)}\left(\cos \left(t G_{2}(\xi)-x \xi\right) \cdot \sum_{k=1}^{n} b_{k} \cos \left(h_{k} \xi\right)-\sin \left(t G_{2}(\xi)-x \xi\right) \cdot \sum_{k=1}^{n} b_{k} \sin \left(h_{k} \xi\right)\right) \\
& =-e^{-t G_{1}(\xi)} \sum_{k=1}^{n} b_{k}\left(\cos \left(t G_{2}(\xi)-x \xi\right) \cdot \cos \left(h_{k} \xi\right)-\sin \left(t G_{2}(\xi)-x \xi\right) \cdot \sin \left(h_{k} \xi\right)\right) \\
& =-e^{-t G_{1}(\xi)} \sum_{k=1}^{n} b_{k} \cos \left(t G_{2}(\xi)-x \xi+h_{k} \xi\right)=-\sum_{k=1}^{n} b_{k} F\left(x-h_{k}, t ; \xi\right)
\end{aligned}
$$


and this proves the theorem for the family of functions $F(x, t ; \xi)$ for each real value of the parameter $\xi$.

Similarly, we are going to check that function (2.8) satisfies equation (1.1) at each point in the half-plane $(x, t) \in \mathbb{R}^{1} \times(0,+\infty)$. We calculate:

$$
\begin{aligned}
H_{x}(x, t ; \xi)= & -\xi e^{-t G_{1}(\xi)} \cos \left(t G_{2}(\xi)-x \xi\right), \\
H_{x x}(x, t ; \xi)= & -\xi^{2} e^{-t G_{1}(\xi)} \sin \left(t G_{2}(\xi)-x \xi\right), \\
H_{t}(x, t ; \xi)= & -G_{1}(\xi) e^{-t G_{1}(\xi)} \sin \left(t G_{2}(\xi)-x \xi\right)+G_{2}(\xi) e^{-t G_{1}(\xi)} \cos \left(t G_{2}(\xi)-x \xi\right), \\
H_{t t}(x, t ; \xi)= & \left(G_{1}^{2}(\xi)-G_{2}^{2}(\xi)\right) e^{-t G_{1}(\xi)} \sin \left(t G_{2}(\xi)-x \xi\right) \\
& -2 G_{1}(\xi) G_{2}(\xi) e^{-t G_{1}(\xi)} \cos \left(t G_{2}(\xi)-x \xi\right) \\
= & -\left(a^{2} \xi^{2}+\sum_{k=1}^{n} b_{k} \cos \left(h_{k} \xi\right)\right) e^{-t G_{1}(\xi)} \sin \left(t G_{2}(\xi)-x \xi\right) \\
& -\sum_{k=1}^{n} b_{k} \sin \left(h_{k} \xi\right) \cdot e^{-t G_{1}(\xi)} \cos \left(t G_{2}(\xi)-x \xi\right) .
\end{aligned}
$$

Substituting the derivatives $H_{t t}$ and $H_{x x}$ into equation (1.1), we obtain

$$
\begin{aligned}
H_{t t}(x, t ; \xi)-a^{2} H_{x x}(x, t ; \xi)= & -e^{-t G_{1}(\xi)}\left(\sin \left(t G_{2}(\xi)-x \xi\right) \cdot \sum_{k=1}^{n} b_{k} \cos \left(h_{k} \xi\right)\right. \\
& \left.+\cos \left(t G_{2}(\xi)-x \xi\right) \cdot \sum_{k=1}^{n} b_{k} \sin \left(h_{k} \xi\right)\right) \\
= & -e^{-t G_{1}(\xi)} \sum_{k=1}^{n} b_{k}\left(\sin \left(t G_{2}(\xi)-x \xi\right) \cdot \cos \left(h_{k} \xi\right)\right. \\
& \left.+\cos \left(t G_{2}(\xi)-x \xi\right) \cdot \sin \left(h_{k} \xi\right)\right) \\
= & -e^{-t G_{1}(\xi)} \sum_{k=1}^{n} b_{k} \sin \left(t G_{2}(\xi)-x \xi+h_{k} \xi\right) \\
= & -\sum_{k=1}^{n} b_{k} H\left(x-h_{k}, t ; \xi\right) .
\end{aligned}
$$

Corollary 2.1. Under condition (1.2), the family of functions

$$
G(x, t ; \alpha, \beta, \xi):=\alpha e^{-t G_{1}(\xi)} \cos \left(t G_{2}(\xi)-x \xi\right)+\beta e^{-t G_{1}(\xi)} \sin \left(t G_{2}(\xi)-x \xi\right),
$$

where $G_{1}(\xi)$ and $G_{2}(\xi)$ are defined by identities (2.5), satisfies equation (1.1) in the classical sense for all real values of the parameters $\alpha, \beta$ and $\xi$.

\section{Classes of Equations SATISFying THE ASSUMPtions OF THEOREM}

Let us find out what relations should be satisfied by the coefficients $a, b_{k}$ and the shifts $h_{k}$, $k=\overline{1, n}$ to ensure condition 1.2 for each real $\xi$. Letting $\xi=0$ in 1.2 we obviously obtain an inequality

$$
\sum_{k=1}^{n} b_{k}>0
$$


We consider a function $a^{2} \xi^{2}+\sum_{k=1}^{n} b_{k} \cos \left(h_{k} \xi\right)$ as $\xi \in(0,+\infty)$. The derivative of this function is equal to

$$
2 a^{2} \xi-\sum_{k=1}^{n} b_{k} h_{k} \sin \left(h_{k} \xi\right)=2 a^{2} \xi\left(1-\sum_{k=1}^{n} \frac{b_{k} h_{k}^{2}}{2 a^{2}} \frac{\sin \left(h_{k} \xi\right)}{h_{k} \xi}\right)
$$

Since

$$
\frac{\sin (h \xi)}{h \xi}<1 \quad \text { for each } \xi \in(0,+\infty)
$$

the inequality holds:

$$
1-\sum_{k=1}^{n} \frac{b_{k} h_{k}^{2}}{2 a^{2}} \frac{\sin \left(h_{k} \xi\right)}{h_{k} \xi}>1-\sum_{k=1}^{n} \frac{b_{k} h_{k}^{2}}{2 a^{2}},
$$

and this implies that the derivative is positive on the segment $\xi \in(0,+\infty)$ under the condition:

$$
2 a^{2} \geqslant \sum_{k=1}^{n} b_{k} h_{k}^{2}
$$

Then the function $a^{2} \xi^{2}+\sum_{k=1}^{n} b_{k} \cos \left(h_{k} \xi\right)$ increases as $\xi \in(0,+\infty)$, while for $\xi \in[0,+\infty)$ it attains its smallest value, which is equal to $\sum_{k=1}^{n} b_{k}>0$. By the evenness of the function, this value is the smallest for all real $\xi \in(-\infty,+\infty)$. Thus, we have shown that condition (1.2), under which the theorem holds, is satisfied if the coefficients $a, b_{k}$ and the shift $h_{k}, k=\overline{1, n}$, obey relations 3.1 and 3.2 .

\section{ACKNOWLEDGMENTS}

The author is deeply grateful to A.B. Muravnik for the formulation of the problem and valuable advices, to A.L. Skubachevsky for a permanent attention to the work, and to the participants of the Second International Scientific conference "Ufa Autumn Mathematical School2020 " for useful discussion of the work.

\section{BIBLIOGRAPHY}

1. J. Bernoulli. Meditationes. Dechordis vibrantibis // Commentaril Academiae Scientiarum Imperialis Petropolitanae. 3, 13-28 (1728).

2. H. Burkhardt. Entwicklungen nach oscillirenden Funktionen und Integration der Differentialgleichungen der mathematischen Physik // Jahresber. Deutsch. Math.-Ver. 10:2, part VI, 1393-1804 (1908).

3. E. Pinney. Ordinary Difference-Differential Equations. University of California press, Berkeley (1958).

4. R. Bellman, K.L. Cooke. Differential-difference equations. Academic Press, New York (1963).

5. A.L. Skubachevskii. Elliptic functional differential equations and applications. Birkhäuser, Basel (1997).

6. A.L. Skubachevskii. Nonclassical boundary value problems. I // Sovrem. Matem. Fundam. Napravl. 26, 3-132 (2007). [J. Math. Sci. 155:2 (2008), 199-334 (2007).]

7. A.L. Skubachevskii. Nonclassical boundary-value problems. II // Sovrem. Matem. Fundam. Napravl. 33, 3-179 (2009). [J. Math. Sci. 166:4, 377-561 (2010).]

8. A.L. Skubachevskii. Boundary-value problems for elliptic functional-differential equations and their applications // Uspekhi Matem. Nauk. 71:5(431), 3-112 (2016). [Russ. Math. Surv. 71:5, 801-906 (2016).] 
9. L.E. Rossovskii. Elliptic functional differential equations with contractions and extensions of independent variables of the unknown function // Sovrem. Matem. Fundam. Napravl. 54, 3-138 (2014). [J. Math. Sci. 223:4, 351-493 (2017).]

10. E.P. Ivanova. On smooth solutions of differential-difference equations with incommensurable shifts of arguments // Matem. Zamet. 105:1, 145-148 (2019). [Math. Notes. 105:1, 140-144 (2019).]

11. A.B. Muravnik. Functional differential parabolic equations: integral transformations and qualitative properties of solutions of the Cauchy problem // Sovrem. Matem. Fundam. Napravl. 52, 3-143 (2014). [J. Math. Sci. 216:3, 345-496 (2016).]

12. A.B. Muravnik. Asymptotic properties of solutions of the Dirichlet problem in the half-plane for differential-difference elliptic equations // Matem. Zamet. 100:4, 566-576 (2016). [Math. Notes. 100:4, 579-588 (2016).]

13. A.B. Muravnik. On the half-plane Dirichlet problem for differential-difference elliptic equations with several nonlocal terms // Math. Model. Nat. Phenom. 12:6, 130-143 (2017).

14. A.B. Muravnik. Elliptic problems with nonlocal potential arising in models of nonlinear optics // Matem. Zamet. 105:5, 747-762 (2019). [Math. Notes. 105:5, 734-746 (2019).]

15. A.B. Muravnik. Half-plane differential-difference elliptic problems with general-kind nonlocal potentials // Compl. Variab. Ellip. Equat., to appear. DOI: 10.1080/17476933.2020.1857372.

16. A.B. Muravnik. Elliptic differential-difference equations in the half-space // Matem. Zamet. 108:5, 764-770 (2020). [Math. Notes. 108:5, 727-732 (2020).]

17. V.V. Vlasov, D.A. Medvedev. Functional-differential equations in Sobolev spaces and related problems of spectral theory // Sovrem. Matem. Fundam. Napravl. 30, 3-173 (2008). [J. Math. Sci. 164:5, 659-841 (2010).]

18. N.V. Zaitseva. On global classical solutions of hyperbolic differential-difference equations // Dokl. RAN. 491:2, 44-46 (2020). [Dokl. Math. 101:2, 115-116 (2020).]

19. N.V. Zaitseva. Global classical solutions of some two-dimensional hyperbolic differential-difference equations // Differ. Uravn. 56:6, 745-751 (2020). [Differ. Equat. 56:6, 734-739 (2020).]

20. N.V. Zaitseva. Classical solutions of hyperbolic differential-difference equations with several nonlocal terms // Lobachevskii J. of Math. 42:1, 231-236 (2021).

21. I.M. Gel'fand, G.E. Shilov. Fourier transforms of rapidly increasing functions and questions of uniqueness of the solution of Cauchy problem // Uspekhi Matem. Nauk. 8:6, 3-54 (1953). (in Russian).

Natalya Vladimirovna Zaitseva,

Lomonosov Moscow State University,

Leninskie gory 1, bld. 52,

119991, Moscow, Russia

E-mail: zaitseva@cs.msu.ru 